\title{
Identification of a nonsense mutation in the PAX9 gene in molar oligodontia
}

\author{
Pekka Nieminen*, $1,2,3$, Sirpa Arte ${ }^{2,3,4}$, Dennis Tanner ${ }^{1}$, Lars Paulin ${ }^{1}$, Satu Alaluusua ${ }^{2,3,4}$, \\ Irma Thesleff ${ }^{1,2,3}$ and Sinikka Pirinen ${ }^{2,3,4}$
}

${ }^{1}$ Institute of Biotechnology, University of Helsinki; ${ }^{2}$ Department of Pedodontics, Institute of Dentistry, University of Helsinki; ${ }^{3}$ Department of Orthodontics, Institute of Dentistry, University of Helsinki; ${ }^{4}$ Department of Oral and Maxillofacial Diseases, Helsinki University Central Hospital, Finland

Development of dentition is controlled by numerous genes, as has been shown by experimental animal studies and mutations that have been identified by genetic studies in man. Here we report a nonsense mutation in the PAX9 gene that is associated with molar tooth agenesis in a Finnish family. The A340T transversion creates a stop codon at lysine 114, and truncates the coded PAX9 protein at the end of the DNAbinding paired-box. All the affected members of the family were heterozygous for the mutation. The tooth agenesis phenotype involves all permanent second and third molars and most of the first molars and resembles the earlier reported phenotype that was also associated with a PAX9 mutation. ${ }^{1}$ The phenotype is presumably a consequence of haploinsufficiency of PAX9. In another Finnish family with molar tooth agenesis, we could not find similar sequence changes in PAX9. European Journal of Human Genetics (2001) 9, $743-746$.

Keywords: tooth agenesis; oligodontia; hypodontia; PAX9; nonsense mutation

\section{Introduction}

Agenesis of permanent teeth (hypodontia or oligodontia) is a common phenotypic feature in man. The prevalence of hypodontia of one or a few permanent second premolars or maxillary lateral incisors in otherwise healthy individuals is six to eight per cent, and agenesis of third molars (wisdom teeth) is even more common. ${ }^{2-4}$ The same teeth are most often missing in oligodontia, ie agenesis of several teeth, which is usually associated with syndromes. Apart from third molars, the agenesis of permanent molars is a rather rare occurrence.

Several genes have been implicated in tooth development by gene expression and experimental studies in mouse. ${ }^{5,6}$ In man, some gene mutations associated with tooth agenesis have been identified. These include ectodermal dysplasias and other syndrome phenotypes ${ }^{7}$ but also mutations that cause oligodontia as an isolated trait. Two different muta-

*Correspondence: Pekka Nieminen, Institute of Biotechnology, PO Box 56, 00014 University of Helsinki, Finland. Tel: + 3589 19159353; Fax: + 3589 19159366; E-mail: pekka.nieminen@helsinki.fi Received 23 April 2001; revised 20 June 2001; accepted 31 July 2001 tions in the gene encoding transcription factor MSX1 have been described in families where dominantly inherited oligodontia involves characteristically second premolars and third molars. ${ }^{8,9}$ A frameshift mutation in another transcription factor gene, $P A X 9$, has been identified in a large kindred where dominant oligodontia affects predominantly permanent molars. ${ }^{1,10}$

MSX1 and PAX9 are transcription factors that are expressed in dental mesenchyme after initiation of tooth development in response to epithelial signals. ${ }^{11,12}$ Mouse null mutations have shown that both are indispensable for tooth and craniofacial development. ${ }^{12,13}$ Lack of Pax9 leads also to malformations of limbs and pharyngeal pouch derivatives. ${ }^{12}$ Paralogues of PAX9 in the family of 'pairedbox' transcription factors have important roles in the development of many organs (for review ${ }^{14}$ ). Interestingly, while the mouse null mutations of several genes including Ms 1 and $\operatorname{Pax} 9$ cause a complete lack of teeth, the mouse heterozygotes usually have normal dentition. This is probably explained by the simplicity of mouse dentition, and emphasizes the relevance of identifying the gene defects in different cases of human tooth agenesis. 
In this report we describe two Finnish familial cases of oligodontia involving permanent molars. In these families, tooth agenesis is inherited in autosomal dominant manner. In one of the families, all affected members carry a nonsense mutation in the paired box of the PAX9 gene.

\section{Materials and methods}

The proband of family 1 was originally sent for treatment to the Paediatric Dental Department of Hospital for Children and Adolescents, Helsinki University Central Hospital. The proband of family 2 was an orthodontic patient of the Department of Pedodontics and Orthodontics, Institute of Dentistry, University of Helsinki. The study was conducted under consent of all family members and approved by the Ethics Committee of the Institute of Dentistry. All family members were examined clinically by one of the authors and agenesis of teeth was also verified with panoramic radiographs.

DNA was extracted from venous blood samples using standard methods. Four sets of primers were used to amplify the coding region of the PAX9 gene (EMBL accession numbers for PAX9 mRNA are X92850 and L09745, and for exons HSA238381, HSA238382, and HSA238383). Amplification was performed in $100 \mu \mathrm{l}$ with DNA concentration of $1.5 \mathrm{ng} / \mu \mathrm{l}, 1.5 \mathrm{mM} \mathrm{MgCl}_{2}$ and $1 \mathrm{mU}$ Dynazyme ${ }^{\mathrm{TM}}$ EXT polymerase (Finnzymes, Espoo, Finland). The primers and PCR conditions were as follows (numbering indicates the position of the first base of the primer in the coding sequence): exon 2, forward primer 1 AGGCAGCTGTCCCAAGCAGCG (exon start - 58), reverse primer 1 GGAGGGCACATTGTACTTGTCGC (357), annealing T $64^{\circ} \mathrm{C}, 32$ cycles; exon 2, forward primer 2 ATCCGACCGTGTGACATCAGCC (109), reverse primer 2 GAGCCCCTACCTTGGTCGGTG (exon end +10 ), annealing $\mathrm{T} 64^{\circ} \mathrm{C}, 30$ cycles; exon 3 , forward primer GGGAGTAAAACTTCACCAGGC (exon start -197), reverse primer CCACCTGGCCTGACCCTC (exon end +28), annealing $\mathrm{T} 61^{\circ} \mathrm{C}$, 32 cycles; exon 4, forward primer GGAGAGTAGAGTCAGAGCATTGCTG (exon start -121), reverse primer GAGACCTGGGAATTGGGGGA (stop +74), annealing $\mathrm{T} 61^{\circ} \mathrm{C}, 32$ cycles. Primers for exons 2 and 4 were adapted from Stockton et al. ${ }^{1}$ PCR products were purified by agarose gel electrophoresis and Qiaquick gel extraction kit (Qiagen, Bothell, WA, USA). Both strands of the PCR products were sequenced using the BigDye terminator chemistry and analysed on an ABI 377XXL DNA sequencer (Applied Biosystems, Foster City, CA, USA).

\section{Results}

Clinical evaluation of the family members did not reveal any significant medical problems except for the lack of several teeth (oligodontia) in three individuals in one family and two in the other family (Table 1 and Figure 1a). The rest of the family members had normal dentition. The affected indivi- duals in family 1 lacked all second and third permanent molars as well as both maxillary lateral incisors (the proband was too young for the definitive diagnosis of the missing third molars to be made). The proband and his mother also lacked all first permanent molars and several second premolars. In the primary dentition of the proband all second molars were missing. The affected brother had all primary teeth but the second primary molars were submerged. The mother and the affected brother had a malposition of upper permanent canine. In addition, some permanent teeth appeared smaller than normal in affected patients. The oligodontia phenotype was more severe in family 2. The proband lacked eight permanent molars, including all third molars, and several other permanent teeth. The affected father had only three permanent teeth: upper central incisors and one lower lateral incisor. Both of them had all primary teeth. The small size of the families does not allow complete segregation analyses to be made, but occurrence of oligodontia in families fits into an autosomal dominant mode of inheritance (Figure 1b).

Sequencing of the PAX9 gene (exons 2, 3 and 4 that include the coding region) revealed three nucleotide changes as compared to the published sequences ${ }^{15-17}$. All changes could be verified from both strands. A nucleotide transversion A340T present in heterozygous state of exon 2 in all

Table 1 Oligodontia phenotypes

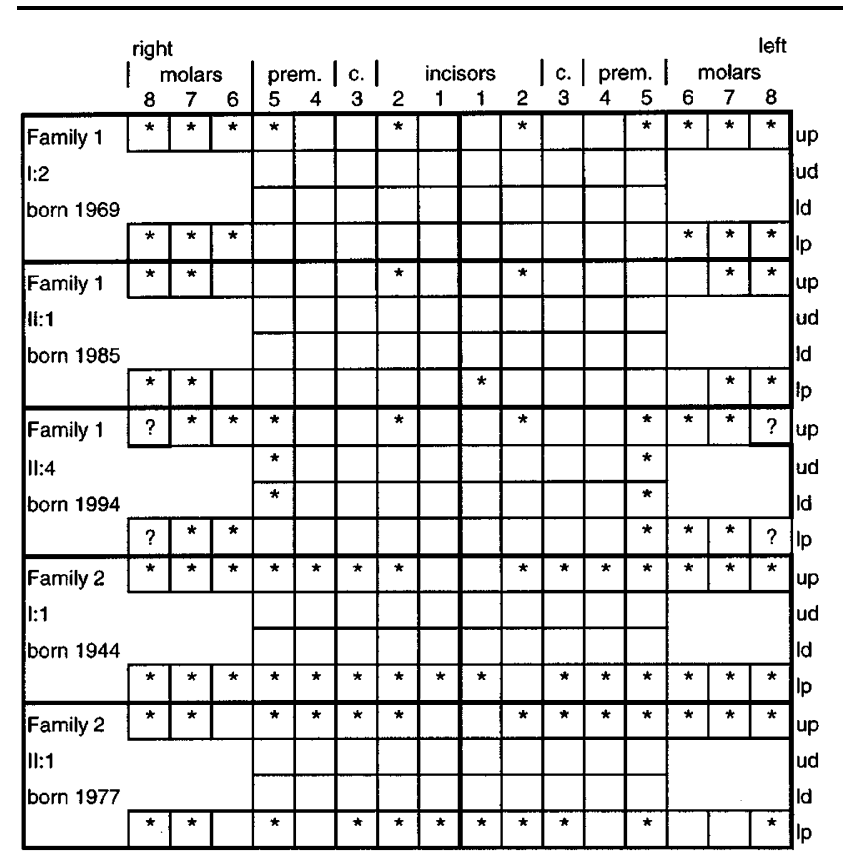

\footnotetext{
*, tooth is missing; ?, diagnosis not possible (patient too young); prem., premolars; c., canines; up, upper permanent teeth; ud, upper deciduous (primary) teeth; ld, lower deciduous (primary) teeth; lp, lower permanent teeth.
} 


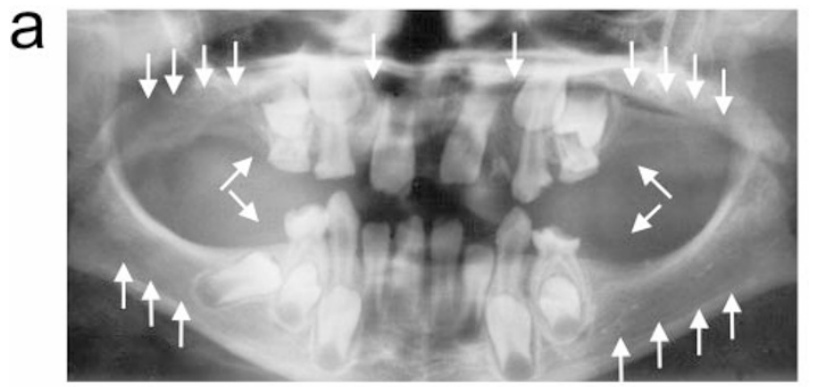

b
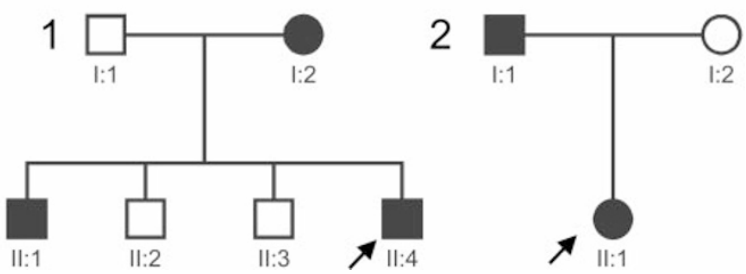

Figure 1 Oligodontia phenotype and families (a) Panoramic radiograph of proband of family 1 (II:4) in age of 6 . Missing teeth are depicted with arrows. (b) Pedigrees of molar oligodontia families 1 and 2. Squares, males; circles, females; darkened, affected; arrows, probands.

three affected members of family 1 produces a stop codon at lysine 114 in the end of the DNA-binding 'paired box' of PAX9 (Figure 2). The premature termination of translation creates a truncated protein that lacks the last $\alpha$-helix $(\alpha 6)$ of the paired box and the entire C-terminal region.

The other nucleotide changes found were in exon 3 , and interestingly, in adjacent nucleotides. The silent change C717T in the codon of His239 was present in heterozygous state in the healthy mother and affected daughter of family 2. A G718C transversion causes a conservative change Ala240Pro. The latter change was present in heterozygous state in all members of family 2 and in homozygous or heterozygous state in some of the members of family 1.

\section{Discussion}

In this paper we report a second inactivating mutation in PAX9 that is associated with oligodontia. Our study was inspired by the similarity of the phenotypes in our families and the American family described earlier. ${ }^{1,10}$ The similarities include the complete lack of second and third permanent molars in all affected members of the families, partial lack of permanent first molars and second premolars as well as the reduction in size of some of the teeth present. In the two families with PAX9 mutations, second premolars were missing only when a neighbouring first molar was absent. In the Finnish family, also some primary teeth were missing, suggesting that the phenotype may be more severe than in the American family. Also, in this family the missing incisors

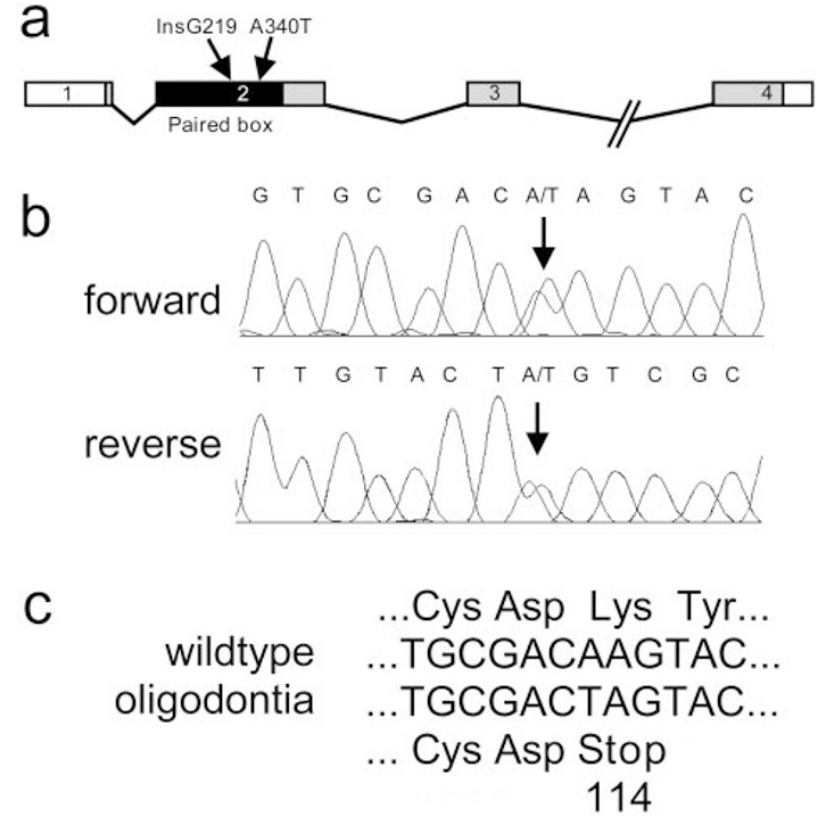

Figure 2 Detection of PAX9 mutation (a) PAX9 gene and protein structure. Boxes delineate exons and gray boxes the coding sequence. Locations of the frameshift mutation from Stockton et $a l^{1}$ and the nonsense mutation described here are depicted. (b) Chromatograms from sequencings of exon 2 of proband of family 1 (II:4). Arrows indicate the heterozygous mutations. (c) Comparison of nucleotide and amino acid sequences of the wild type and mutant genes and proteins.

were all maxillary, whereas in the American family they were mandibular. Similar difference, even though not so complete, applied also to missing premolars. This difference may be attributed to unknown modifying genetic factors, the effect of which can recurrently be seen as a variation of patterns of missing teeth.

The A340T mutation creates a stop codon and truncates the PAX9 protein in the C-terminal subdomain of the DNAbinding paired box. The insG219 mutation described by Stockton et al, ${ }^{1}$ destroys the paired box 121 nucleotides earlier, between the $\mathrm{N}$ - and C- subdomains. ${ }^{18}$ The lack of the C-terminal region that follows the paired box most probably disrupts the normal function of both mutant proteins. The mildness of the phenotypes as compared to the PAX9 mouse null mutant suggests that both mutations are loss-of-function and exert their effect mainly through inactivation of one copy of protein, leading to haploinsufficiency. It is possible that the mutant proteins retain some of the DNA binding capacity, and especially the slightly more severe phenotype of family 1 may be attributed to the dominant negative effect due to the more complete paired box of the Lys114Stop mutant protein.

The identification of a second mutation in the PAX9 gene that segregates with oligodontia of molar teeth supports the idea that $P A X 9$ is especially important for 
the development of the most distal teeth, ie molars. Instead, the consensus phenotype for a MSX1 haplosufficiency includes agenesis of second premolars and third molars and in only some cases of first and second molars. ${ }^{8,9}$ In mouse, both Pax 9 and Msx 1 are induced in dental mesenchyme in response to epithelial signals, and subsequently regulate the reciprocal signals from the mesenchyme (for review ${ }^{5,19}$ ). Both genes are also indispensable for tooth organogenesis, as shown by mouse null mutants where tooth development is arrested at bud stage. ${ }^{12,13}$ The differences in the consequences of the haplosufficiency most probably reflect the differing relative importance of the two genes in the development of different tooth families (incisors, premolars and molars). In different types of hypodontia or oligodontia, those teeth in each tooth family that develop latest are most vulnerable for agenesis. It is believed that agenesis follows from inability to overcome a critical threshold at an early stage of development. The MSX1 haploinsufficiency appears to affect all tooth families, while reduced amount of PAX9 protein may be especially critical for molars. As lack of mouse Pax9 leads to decreased expression of $M s \times 1,{ }^{12}$ it is possible that the effect of PAX9 haploinsufficiency on some incisors and premolars are realized through reduced expression of MSX1.

Our inability to find a coding region mutation in the other Finnish family described here may be attributed either to a presence of a mutation in the non-coding regulatory regions of the $P A X 9$ gene, or a mutation in another gene, perhaps acting upstream or downstream of $P A X 9$. In our work with a milder phenotype, where only a few premolars or incisors are missing, we have detected recombinations both with respect to MSX1 and $P A X 9^{4}$ (Nieminen et al, manuscript in preparation). However, these milder phenotypes may also be caused by genes acting in the same signaling pathways with MSX1 and PAX9.

\section{Acknowledgements}

We would like to thank the families for their participation in the study. This work was supported by the Academy of Finland. D Tanner was a participant of the U.S. Minority International Research Program in Molecular Biology.

\section{References}

1 Stockton DW, Das P, Goldenberg M, D'Souza RN, Patel PI. Mutation of PAX9 is associated with oligodontia. Nat Genet 2000; 24: $18-19$.

2 Grahnen H. Hypodontia in the permanent dentition. Odont Revy 1956; 7: $1-100$.

3 Haavikko K. Hypodontia of permanent teeth. Proc Finn Dent Soc 1971; 67: 219-225.

4 Arte S, Nieminen P, Apajalahti S, Haavikko K, Thesleff I, Pirinen S: Characteristics of incisor-premolar hypodontia in families. $J$ Dent Res; 80: 1445 - 1450 .

5 Jernvall J, Thesleff I: Reiterative signaling and patterning during mammalian tooth morphogenesis. Mech Dev 2000; 92: 19-29.

6 Nieminen, P, Pekkanen, M, Ảberg, T, Thesleff, I: Gene expression in tooth. Developmental Biology Programme, Institute of Biotechnology, University of Helsinki, Finland, 2001.

7 Online Mendelian Inheritance in Man, OMIM (TM): McKusickNathans Institute for Genetic Medicine, Johns Hopkins University (Baltimore, MD) and National Center for Biotechnology Information, National Library of Medicine (Bethesda, MD), 2001.

8 Vastardis H, Karimbux N, Guthua SW, Seidman JG, Seidman CE: A human MSX1 homeodomain missense mutation causes selective tooth agenesis. Nat Genet 1996; 13: 417-421.

9 van den Boogaard MJH, Dorland M, Beemer FA, van Amstel HKP: MSX1 mutation is associated with orofacial clefting and tooth agenesis in humans. Nat Genet 2000; 24: 342-343.

10 Goldenberg M, Das P, Messersmith $\mathrm{M}$ et al: Clinical, radiographic, and genetic evaluation of a novel form of autosomaldominant oligodontia. J Dent Res 2000; 79: 1469-1475.

11 Vainio S, Karavanova I, Jowett A, Thesleff I: Identification of BMP-4 as a signal mediating secondary induction between epithelial and mesenchymal tissues during early tooth development. Cell 1993; 75: 45-58.

12 Peters H, Neubuser A, Kratochwil K, Balling R: Pax9-deficient mice lack pharyngeal pouch derivatives and teeth and exhibit craniofacial and limb abnormalities. Genes Dev 1998; 12: 2735 2747.

13 Satokata I, Maas R: Msx1 deficient mice exhibit cleft palate and abnormalities of craniofacial and tooth development. Nat Genet 1994; 6: $348-356$.

14 Dahl E, Koseki H, Balling R: Pax genes and organogenesis. Bioessays 1997; 19: $755-765$.

15 Hetzer-Egger C, Schorpp M, Boehm T: Evolutionary conservation of gene structures of the Pax1/9 gene family. Biochim Biophys Acta 2000; 1492: 517 -521.

16 Stapleton P, Weith A, Urbanek P, Kozmik Z, Busslinger M: Chromosomal localization of seven PAX genes and cloning of a novel family member, PAX-9. Nat Genet 1993; 3: 292-298.

17 Peters H, Schuster G, Neubuser A, Richter T, Hofler H, Balling R: Isolation of the Pax9 cDNA from adult human esophagus. Mamm Genome 1997; 8: 62-64.

$18 \mathrm{Xu} \mathrm{HE}$, Rould MA, Xu W, Epstein JA, Maas RL, Pabo CO: Crystal structure of the human Pax6 paired domain-DNA complex reveals specific roles for the linker region and carboxy-terminal subdomain in DNA binding. Genes Dev 1999; 13: 1263-1275.

19 Thesleff, I, Nieminen, P: Tooth induction. Nature Publishing Group, Macmillan Publishers Ltd, 2001. 